\title{
Erratum to: An Efficient Method for Target Extraction of Infrared Images
}

\author{
Ying Ling and Xingjin Mao \\ School of Computer Science Northwestern Polytechnical University \\ Shanxi, Xi' an, 710129, China \\ lybyp@nwpu.edu.cn, Maoxingj1985@gmail.com
}

F.L. Wang et al. (Eds.): AICI 2010, Part I, LNAI 6319, pp. 185-192, 2010.

(C) Springer-Verlag Berlin Heidelberg 2010

DOI 10.1007/978-3-642-16530-6_59

The name of the first author was erroneously printed "Ying Ling". The correct spelling is "Ying Li". 